\title{
Stochastic Nonlinear Complementarity Problem and Applications to Traffic Equilibrium under Uncertainty
}

\author{
C. Zhang $\cdot$ X. Chen
}

Published online: 15 April 2008

(C) Springer Science+Business Media, LLC 2008

\begin{abstract}
The expected residual minimization (ERM) formulation for the stochastic nonlinear complementarity problem (SNCP) is studied in this paper. We show that the involved function is a stochastic $R_{0}$ function if and only if the objective function in the ERM formulation is coercive under a mild assumption. Moreover, we model the traffic equilibrium problem (TEP) under uncertainty as SNCP and show that the objective function in the ERM formulation is a stochastic $R_{0}$ function. Numerical experiments show that the ERM-SNCP model for TEP under uncertainty has various desirable properties.
\end{abstract}

Keywords Stochastic nonlinear complementarity problem · Expected residual minimization · Traffic equilibrium problem under uncertainty $\cdot$ Stochastic $R_{0}$ function

\section{Introduction}

In this paper, we consider the stochastic nonlinear complementarity problem

$$
x \geq 0, \quad F(x, \omega) \geq 0, \quad x^{T} F(x, \omega)=0, \quad \omega \in \Omega,
$$

Communicated by M. Fukushima.

This work was partially supported by a Grant-in-Aid from the Japan Society for the Promotion of Science. The authors thank Professor Guihua Lin for pointing out an error in Proposition 2.1 on an earlier version of this paper. The authors are also grateful to the referees for their insightful comments.

C. Zhang

Department of Applied Mathematics, Beijing Jiaotong University, Beijing, China

X. Chen $(\varangle)$

Department of Applied Mathematics, The Hong Kong Polytechnic University, Hong Kong,

Hong Kong

e-mail: maxjchen@polyu.edu.hk 
where $\omega \in \Omega \subseteq R^{m}$ is a random vector with given probability distribution $\mathcal{P}$ and $F: R^{n} \times \Omega \rightarrow R^{n}$ is a given vector-valued function. We denote problem (1) by $\operatorname{SNCP}(F(x, \omega))$.

If $\Omega$ is a singleton, $\operatorname{SNCP}(F(x, \omega))$ reduces to the intensively studied nonlinear complementarity problem; see the comprehensive books ([1] and [2]) for theoretical analysis, numerical algorithms and applications especially in economics and engineering. In reality, due to stochastic factors, the function value of $F$ depends not only on the variables $x$, but also on random vectors. The SNCP provides a framework for modeling of equilibria under uncertainty as a special case of stochastic variational inequalities. Recently, Lin and Fukushima [3] reformulated the SNCP as a stochastic mathematical programming problem with equilibrium constraints. When $F$ is an affine function of $x$ for any $\omega \in \Omega$,

$$
F(x, \omega)=M(\omega) x+q(\omega), \quad \omega \in \Omega,
$$

where $M(\omega) \in R^{n \times n}$ and $q(\omega) \in R^{n}$, the $\operatorname{SNCP}(F(x, \omega))$ reduces to the stochastic linear complementarity problem (SLCP), denoted by $\operatorname{SLCP}(M(\omega), q(\omega))$, which has been studied recently in [4-6].

The expected value (EV) formulation introduced in [7] and the expected residual minimization (ERM) introduced in [4] are two deterministic formulations for the SNCP. The EV formulation is to solve a single nonlinear complementarity problem $\operatorname{NCP}(E[F(x, \omega)])$. The ERM formulation is to minimize the expected residual of the $\operatorname{NCP}(F(x, \omega))$ for all $\omega \in \Omega$. A version of the ERM formulation using NCP functions is to find an optimal solution of

$$
\min _{x \in R_{+}^{n}} f(x):=E\left[\|\Phi(x, \omega)\|^{2}\right],
$$

where

$$
\Phi(x, \omega)=\left(\phi\left(F_{1}(x, \omega), x_{1}\right), \ldots, \phi\left(F_{n}(x, \omega), x_{n}\right)\right),
$$

and $\phi: R^{2} \rightarrow R$ is an NCP function, which satisfies

$$
\phi(a, b)=0 \quad \Longleftrightarrow \quad a \geq 0, b \geq 0, a b=0 .
$$

Many NCP functions have been studied for solving nonlinear complementarity problems [2]. In this paper, we study the ERM formulation (3) for SNCP with the following three NCP functions:

(i) The min function $\phi_{1}(a, b)=\min (a, b)$.

(ii) The FB function $\phi_{2}(a, b)=a+b-\sqrt{a^{2}+b^{2}}$.

(iii) The penalized FB function $\phi_{3}(a, b)=\lambda \phi_{2}(a, b)+(1-\lambda) a_{+} b_{+}, \lambda \in(0,1)$.

It is known ([5] and [8]) that there exist positive constants $c_{1}, c_{2}, c_{3}$ such that

$$
c_{1}\left|\phi_{1}(a, b)\right| \leq c_{2}\left|\phi_{2}(a, b)\right| \leq c_{3}\left|\phi_{1}(a, b)\right| \leq\left|\phi_{3}(a, b)\right| .
$$

The above relation indicates that $\phi_{1}$ and $\phi_{2}$ have the same growth rate, and the growth rate of $\phi_{3}$ is no less than that of $\phi_{1}$ and $\phi_{2}$. In the following, we use $f_{i}$ to distinct $f$ defined by $\phi_{i}$ for $i=1,2,3$, and $f$ when we study their common properties. 
In this paper, we study the solution set of the ERM formulation (3) for the SNCP. In particular, we define a stochastic $R_{0}$ function and show that $F$ is a stochastic $R_{0}$ function if and only if the objective function $f_{1}$ in the ERM formulation (3) for the $\operatorname{SNCP}(F(x, \omega))$ is coercive, i.e., $f_{1}(x) \rightarrow \infty$ as $\|x\| \rightarrow \infty$, under a mild assumption. Moreover, we model the traffic equilibrium problem (TEP) under uncertainty as SNCP and show that the involved function $F$ is a stochastic $R_{0}$ function. Our numerical experiments show that a solution of the ERM formulation has high reliability and delivered rate.

The NCP model with effective algorithms for static TEP based on the Wardrop equilibrium principle [9] has been widely studied ([2] and [10-12]). On the other hand, disruptive events such as uncertain demands, adverse weather, road construction, traffic accidents, landslides, earthquakes, may disrupt greatly one static equilibrium of a network. Recently, Fernando and Nichlàs [13] address this problem and extend the Wardrop equilibrium principle to TEP under uncertainty by defining a robust Wardrop equilibrium (RWE). Their equilibria is supposed to be robust in the sense that it has optimal worst-case cost, which is different with the robustness of SNCP c.f. [5].

The remainder of this paper is organized as follows: In Sect. 2, we introduce the concepts of a stochastic $R_{0}$ function, and equicoercivity. We show that under the assumption that $F$ is equicoercive, $F$ being a stochastic $R_{0}$ function is a necessary and sufficient condition for the coercivity of $f_{1}$ in the ERM formulation. In Sect. 3, we model the TEP under uncertainty as a stochastic $R_{0}$ function NCP. In Sect. 4, we report numerical results of the ERM formulation and the EV formulation for TEP under uncertainty.

We will use the following notations. $\langle l, u\rangle$ represents the set $\{l, l+1, \ldots, u\}$ for natural numbers $l$ and $u$ with $l<u, z_{+}=\max (z, 0)$ for any given vector $z,|\mathcal{S}|$ denotes the cardinality of a given finite set $\mathcal{S}$, and $\|\cdot\|$ refers to the Euclidean norm. Given a set $\Omega \subseteq R^{m}$ of random vectors, let $\operatorname{supp} \Omega$ be the support set of $\Omega$. For a given subset $\hat{\Omega} \subseteq \Omega$ and a function $s: \Omega \rightarrow R_{+}$, we use $E_{\hat{\Omega}}[s(\omega)]$ to represent $E\left[s(\omega) 1_{\{\omega \in \hat{\Omega}\}}\right]$ for simplicity, where $1_{\{\omega \in \hat{\Omega}\}}$ is the indicator function of the set $\hat{\Omega}$, which is equal to 1 if $\omega \in \hat{\Omega}$ and 0 if $\omega \in \Omega \backslash \hat{\Omega}$. Throughout the paper, we suppose the following assumption holds:

Assumption A1 $F(\cdot, \omega)$ is a continuous function for $\omega \in \Omega$ a.e. and $E\left[\|F(x, \omega)\|^{2}\right]$ $<\infty$ at any $x \in R_{+}^{n}$.

Remark 1.1 Note that $\|\min (x, F(x, \omega))\| \leq\|F(x, \omega)\|$ for any $x \in R_{+}^{n}$. It is easy to verify that $E\left[\|F(x, \omega)\|^{2}\right]<\infty$ at any $x \in R_{+}^{n}$ implies that $f(x)<\infty$ at any $x \in R_{+}^{n}$. Moreover, from Proposition 1 in Chap. 2 [14], if there exists a function $z(\omega)$ such that $\|F(x, \omega)\|^{2} \leq z(\omega)$ a.e. for all $x$ in a neighborhood of $\hat{x}$, and $E[z(\omega)]<\infty$, then $f$ is continuous at $\hat{x}$ under Assumption A1.

\section{Solution Set of ERM for SNCP}

In this section we investigate solvability of the ERM formulation for the SNCP. We define a stochastic $R_{0}$ function. Under the assumption that $F$ is equicoercive, we 
prove that the involved function being a stochastic $R_{0}$ function is a necessary and sufficient condition for the coercivity of the objective function in the ERM formulation.

The solution set of the ERM formulation for the SLCP has been studied in [4-6]. Some results depending on the special affine construction of $F(x, \omega)$ in the SLCP cannot be simply generalized to the SNCP. For instance, Lemma 2.2 in [5] states that the ERM formulation for the $\operatorname{SLCP}(M(\omega), q(\omega))$ defined by the 'min' function always has a solution when $\Omega$ is composed of finite elements. However, the following example tells us that we do not have the same result for the $\operatorname{SNCP}(F(x, \omega))$.

Example 2.1 Let $F(x, \omega)=\left(\frac{1}{2}-\frac{3}{2} \omega\right) e^{-\frac{\omega}{2} x}-\omega$ where $\omega \in \Omega=\left\{\omega^{1}, \omega^{2}\right\}$. Here, $\omega^{1}=0, \omega^{2}=1$, and $\mathcal{P}\left\{\omega^{1}\right\}=\mathcal{P}\left\{\omega^{2}\right\}=\frac{1}{2}$. Then the objective function in the ERM formulation for $\operatorname{SNCP}(F(x, \omega))$ defined by $\phi_{1}$ is

$$
\begin{aligned}
f_{1}(x) & =\frac{1}{2}\left\|\min \left(x, \frac{1}{2}\right)\right\|^{2}+\frac{1}{2}\left\|\min \left(x,-e^{-\frac{x}{2}}-1\right)\right\|^{2} \\
& = \begin{cases}\frac{1}{2} x^{2}+\frac{1}{2}\left(e^{-x}+1+2 e^{-\frac{x}{2}}\right) & x \in\left[0, \frac{1}{2}\right], \\
\frac{1}{8}+\frac{1}{2}\left(e^{-x}+1+2 e^{-\frac{x}{2}}\right) & x \in\left(\frac{1}{2}, \infty\right) .\end{cases}
\end{aligned}
$$

It is easy to find that, for $x \in\left[0, \frac{1}{2}\right]$,

$$
f_{1}(x) \geq \frac{1}{2}\left(e^{-\frac{1}{2}}+1+2 e^{-\frac{1}{4}}\right)=\frac{1}{2}+\frac{1}{2 \sqrt{e}}+e^{-\frac{1}{4}}>\frac{5}{8},
$$

and for $x \in\left(\frac{1}{2}, \infty\right), f_{1}(x)$ is strictly decreasing and tending to $\frac{5}{8}$ as $x$ tends to $\infty$. Hence, the ERM formulation defined by the 'min' function has no solution. Moreover, we have $E[F(x, \omega)]=-\frac{1}{4}-\frac{1}{2} e^{-\frac{x}{2}}<0$ for any $x$. Thus, the EV formulation $\operatorname{NCP}(E[F(x, \omega)])$ has no solution.

However, for any $\lambda \in(0,1)$ and $x \in R_{+}$,

$$
f_{3}(x) \geq \frac{1}{2}\left[\lambda\left(x+\frac{1}{2}-\sqrt{x^{2}+\frac{1}{4}}\right)+\frac{1}{2}(1-\lambda) x\right]^{2} \geq \frac{1}{8}(1-\lambda)^{2} x^{2},
$$

which is coercive, and hence ERM formulation defined by $\phi_{3}$ has a nonempty and bounded solution set.

\subsection{Stochastic $R_{0}$ Function}

The $R_{0}$ property relates closely to the boundedness of level sets in the literature of the complementarity problem. For $\operatorname{NCP}(G), G: R^{n} \rightarrow R^{n}$ is an $R_{0}$ function if and only if the function $\|\min (x, G(x))\|^{2}$ is coercive.

Definition 2.1 (See [2]) The function $G: R^{n} \rightarrow R^{n}$ is called an $R_{0}$ function on a set $\mathcal{D} \subseteq R^{n}$ if, for every infinite sequence $\left\{x^{k}\right\} \subseteq \mathcal{D}$ satisfying

$$
\lim _{k \rightarrow \infty}\left\|x^{k}\right\|=\infty, \quad \limsup _{k \rightarrow \infty}\left\|\left(-x^{k}\right)_{+}\right\|<\infty, \quad \limsup _{k \rightarrow \infty}\left\|\left(-G\left(x^{k}\right)\right)_{+}\right\|<\infty,
$$

there exists $i \in\langle 1, n\rangle$ such that $\limsup _{k \rightarrow \infty} \min \left(x_{i}^{k}, G_{i}\left(x^{k}\right)\right)=\infty$. 
Now, we define a stochastic $R_{0}$ function.

Definition 2.2 $F: R^{n} \times \Omega \rightarrow R^{n}$ is called a stochastic $R_{0}$ function on a set $\mathcal{D} \subseteq R^{n}$ if, for every infinite sequence $\left\{x^{k}\right\} \subseteq \mathcal{D}$ satisfying

$$
\begin{aligned}
& \lim _{k \rightarrow \infty}\left\|x^{k}\right\|=\infty, \quad \limsup _{k \rightarrow \infty}\left\|\left(-x^{k}\right)_{+}\right\|<\infty, \\
& \limsup _{k \rightarrow \infty}\left\|\left(-F\left(x^{k}, \omega\right)\right)_{+}\right\|<\infty \quad \text { a.e. }
\end{aligned}
$$

there exists $i \in\langle 1, n\rangle$ such that $\mathcal{P}\left\{\omega: \lim \sup _{k \rightarrow \infty} \min \left(x_{i}^{k}, F_{i}\left(x^{k}, \omega\right)\right)=\infty\right\}>0$.

If $\Omega$ is a singleton, Definition 2.2 reduces to Definition 2.1 .

Definition 2.3 We say $F: R^{n} \times \Omega \rightarrow R^{n}$ is equicoercive on $\mathcal{D} \subseteq R^{n}$ if, for any $\left\{x^{k}\right\} \subseteq \mathcal{D}$ satisfying $\left\|x^{k}\right\| \rightarrow \infty$, the existence of $\left\{\omega^{k}\right\} \subseteq$ supp $\Omega$ with $\lim _{k \rightarrow \infty} F_{i}\left(x^{k}, \omega^{k}\right)=\infty\left(\lim _{k \rightarrow \infty}\left(-F_{i}\left(x^{k}, \omega^{k}\right)\right)_{+}=\infty\right)$ for some $i \in\langle 1, n\rangle$ implies that there exists $\left\{x^{k_{j}}\right\} \subseteq\left\{x^{k}\right\}$ such that

$$
\mathcal{P}\left\{\omega: \lim _{k_{j} \rightarrow \infty} F_{i}\left(x^{k_{j}}, \omega\right)=\infty\right\}>0\left(\mathcal{P}\left\{\omega: \lim _{k_{j} \rightarrow \infty}\left(-F_{i}\left(x^{k_{j}}, \omega\right)\right)_{+}=\infty\right\}>0\right) .
$$

Proposition 2.1 $F: R^{n} \times \Omega \rightarrow R^{n}$ is equicoercive if $\Omega$ is a compact set and there exist constants $L>0$ and $\delta>0$ such that, if $\left\|\omega^{1}-\omega^{2}\right\|<\delta$, then

$$
\left\|F\left(x, \omega^{1}\right)-F\left(x, \omega^{2}\right)\right\|<L, \quad \text { for any } x \in R_{+}^{n} .
$$

Proof We only consider the case that $\left\{x^{k}\right\} \subseteq \mathcal{D}$ with $\left\|x^{k}\right\| \rightarrow \infty$, and $\left\{\omega^{k}\right\} \subseteq \operatorname{supp} \Omega$ satisfy $\lim _{k \rightarrow \infty} F_{i}\left(x^{k}, \omega^{k}\right)=\infty$. For the case $\lim _{k \rightarrow \infty}\left(-F_{i}\left(x^{k}, \omega^{k}\right)\right)_{+}=\infty$, it can be proved in the similar way.

Since $\Omega$ is a compact set and $\operatorname{supp} \Omega$ is a closed set, $\left\{\omega^{k}\right\}$ has an accumulation point $\bar{\omega} \in \operatorname{supp} \Omega$. Let $\left\{\omega^{k_{j}}\right\} \subseteq\left\{\omega^{k}\right\}$ be a subsequence converging to $\bar{\omega}$. It is clear that there is $K>0$ such that $\left\|\omega^{k_{j}}-\bar{\omega}\right\|<\delta$ for any $k_{j} \geq K$. Thus, for any $\|\omega-\bar{\omega}\|<\delta$ and $k_{j} \geq K$,

$$
\begin{aligned}
\left|F_{i}\left(x^{k_{j}}, \omega\right)-F_{i}\left(x^{k_{j}}, \omega^{k_{j}}\right)\right| \leq & \left\|F\left(x^{k_{j}}, \omega\right)-F\left(x^{k_{j}}, \omega^{k_{j}}\right)\right\| \\
\leq & \left\|F\left(x^{k_{j}}, \omega\right)-F\left(x^{k_{j}}, \bar{\omega}\right)\right\| \\
& +\left\|F\left(x^{k_{j}}, \bar{\omega}\right)-F\left(x^{k_{j}}, \omega^{k_{j}}\right)\right\| \\
< & 2 L,
\end{aligned}
$$

which implies $\lim _{k_{j} \rightarrow \infty} F_{i}\left(x^{k_{j}}, \omega\right)=\infty$. Hence,

$$
\mathcal{P}\left\{\omega: \lim _{k_{j} \rightarrow \infty} F_{i}\left(x^{k_{j}}, \omega\right)=\infty\right\} \geq \mathcal{P}\{\omega \in \Omega:\|\omega-\bar{\omega}\|<\delta\}>0 .
$$

Therefore, $F$ is equicoercive. 
Remark 2.1 If $\Omega$ has only finite elements, or $F$ is uniformly continuous with respect to $\omega \in \Omega$ on $R_{+}^{n}$, the condition of Proposition 2.1 holds.

From Definitions 2.1-2.3, we can easily get the following proposition.

Proposition 2.2 Suppose that $F(\cdot, \bar{\omega})$ is an $R_{0}$ function on a set $\mathcal{D}$ for some $\bar{\omega} \in$ $\operatorname{supp} \Omega$, and $F$ is equicoercive on $\mathcal{D}$, then $F$ is a stochastic $R_{0}$ function on $\mathcal{D}$.

Proof Suppose that $\left\{x^{k}\right\} \subseteq \mathcal{D}$ satisfies (6). If $\limsup _{k \rightarrow \infty}\left\|\left(-F\left(x^{k}, \bar{\omega}\right)\right)_{+}\right\|=\infty$, then there exist $i \in\langle 1, n\rangle$ and $\left\{x^{\tilde{k}_{j}}\right\} \subseteq\left\{x^{k}\right\}$ such that $\lim _{\tilde{k}_{j} \rightarrow \infty}\left(-F_{i}\left(x^{\tilde{k}_{j}}, \bar{\omega}\right)\right)_{+}=\infty$. By using the assumption that $F$ is equicoercive, there exists $\left\{x^{k_{j}}\right\} \subseteq\left\{x^{\tilde{k}_{j}}\right\}$ such that

$$
\mathcal{P}\left\{\omega: \lim _{k_{j} \rightarrow \infty}\left(-F_{i}\left(x^{k_{j}}, \omega\right)\right)_{+}=\infty\right\}>0,
$$

which contradicts to the fact that $\limsup _{k \rightarrow \infty}\left\|\left(-F\left(x^{k}, \omega\right)\right)_{+}\right\|<\infty$ a.e. in (6). Hence $\limsup _{k \rightarrow \infty}\left\|\left(-F\left(x^{k}, \bar{\omega}\right)\right)_{+}\right\|<\infty$. Since $F(\cdot, \bar{\omega})$ is an $R_{0}$ function, there exists $i \in\langle 1, n\rangle$ such that

$$
\limsup _{k \rightarrow \infty} \min \left(x_{i}^{k}, F_{i}\left(x^{k}, \bar{\omega}\right)\right)=\infty .
$$

Using the assumption that $F$ is equicoercive again, we obtain that

$$
\mathcal{P}\left\{\omega: \limsup _{k \rightarrow \infty} \min \left(x_{i}^{k}, F_{i}\left(x^{k}, \omega\right)\right)=\infty\right\}>0 .
$$

Therefore, $F$ is a stochastic $R_{0}$ function on the set $\mathcal{D}$.

We use Example 3.2 in [15] to show that the assumption of equicoercivity in Proposition 2.2 cannot be omitted.

Example 2.2 Let $\omega \in \Omega=[-2,2]$, where $\omega$ is uniformly distributed on $\Omega$. Consider the function $F: R_{+} \times \Omega \rightarrow R$ defined by

$$
F(x, \omega):= \begin{cases}2+\omega, & \omega \in[-2,0], \\ 2-\omega, & \omega \in(0,2],\end{cases}
$$

for $x \in[0,1]$ and

$$
F(x, \omega):= \begin{cases}2 x+x^{3} \omega, & \omega \in\left[-\frac{2}{x^{2}},-\frac{1}{x^{2}}\right], \\ x+x^{3}+x^{5} \omega, & \omega \in\left(-\frac{1}{x^{2}}, 0\right], \\ x+x^{3}-x^{5} \omega, & \omega \in\left(0, \frac{1}{x^{2}}\right], \\ 2 x-x^{3} \omega, & \omega \in\left(\frac{1}{x^{2}}, \frac{2}{x^{2}}\right], \\ 0, & \omega \in\left[-2,-\frac{2}{x^{2}}\right) \cup\left(\frac{2}{x^{2}}, 2\right],\end{cases}
$$


for $x \in(1, \infty)$. The function $F$ is continuous on $R_{+} \times \Omega$ and Assumption A1 holds. It is easy to check that $F(\cdot, 0)$ is an $R_{0}$ function on $R_{+}$, but $F$ is not a stochastic $R_{0}$ function on $R_{+}$. Moreover, $F$ is not equicoercive on $R_{+}^{n}$, as $\lim _{x^{k} \rightarrow \infty} F\left(x^{k}, 0\right)=\infty$, and $\mathcal{P}\left\{\omega: \lim \sup _{x^{k} \rightarrow \infty} F_{i}\left(x^{k}, \omega\right)=\infty\right\}=0$.

The following example shows that the inverse of Proposition 2.2 does not hold. For a stochastic $R_{0}$ function $F$, even if $F$ is equicoercive, it is not necessary to have that $F(\cdot, \tilde{\omega})$ is an $R_{0}$ function for some $\tilde{\omega} \in \operatorname{supp} \Omega$. Moreover, $E[F(\cdot, \omega)]$ is not necessary to be an $R_{0}$ function.

Example 2.3 Consider the function

$$
F(x, \omega)=\left((-\omega)_{+} e^{x_{1}}, \omega_{+} e^{x_{2}}, \operatorname{sign}(\omega) x_{3}\right),
$$

where $x=\left(x_{1}, x_{2}, x_{3}\right)$ and $\omega$ is uniformly distributed on $\Omega=[-1,1]$. It is not difficult to show that Assumption A1 holds and $F$ is equicoercive. For a fixed $\tilde{\omega} \leq 0$ and a sequence $\left\{x^{k}\right\}$, where $x^{k}=(0, k, 0)$ for $k=1,2, \ldots$, it is easy to verify that $\left\{x^{k}\right\}$ satisfies (5) with $G\left(x^{k}\right)=F\left(x^{k}, \tilde{\omega}\right)=(-\tilde{\omega}, 0,0)$ and

$$
\limsup _{k \rightarrow \infty} \min \left(x_{i}^{k}, G_{i}\left(x^{k}\right)\right)=0, \quad \text { for } i=1,2,3 .
$$

Similarly, for a fixed $\tilde{\omega}>0$ and a sequence $\left\{x^{k}\right\}$ defined by $x^{k}=(k, 0,0)$ for $k=$ $1,2, \ldots,(5)$ holds with $G\left(x^{k}\right)=F\left(x^{k}, \tilde{\omega}\right)=(0, \tilde{\omega}, 0)$, and

$$
\limsup _{k \rightarrow \infty} \min \left(x_{i}^{k}, G_{i}\left(x^{k}\right)\right)=0, \quad \text { for } i=1,2,3 .
$$

Thus, $F(\cdot, \omega)$ is not an $R_{0}$ function for any fixed $\omega \in[-1,1]$. Moreover, we can show that $E[F(x, \omega)]=\left(e^{x_{1}} / 4, e^{x_{2}} / 4,0\right)$ is not an $R_{0}$ function by using a sequence $\left\{x^{k}\right\}$ where $x^{k}=(0,0, k)$ for $k=1,2, \ldots$ However, for every infinite sequence $\left\{x^{k}\right\}$ satisfying (6), if $\limsup _{k \rightarrow \infty} x_{1}^{k}=\infty$, we have

$$
\mathcal{P}\left\{\omega: \limsup _{k \rightarrow \infty} \min \left(x_{1}^{k}, F_{1}\left(x^{k}, \omega\right)\right)=\infty\right\}=\mathcal{P}\{\omega: \omega \in[-1,0)\}=\frac{1}{2} .
$$

If $\limsup _{k \rightarrow \infty} x_{i}^{k}=\infty$ where $i \in\langle 2,3\rangle$, we have

$$
\mathcal{P}\left\{\omega: \limsup _{k \rightarrow \infty} \min \left(x_{i}^{k}, F_{i}\left(x^{k}, \omega\right)\right)=\infty\right\}=\mathcal{P}\{\omega: \omega \in(0,1]\}=\frac{1}{2} .
$$

Therefore, $F$ is a stochastic $R_{0}$ function.

We call $A \in R^{n \times n}$ an $R_{0}$ matrix [1] if

$$
x \geq 0, \quad A x \geq 0, \quad x^{T} A x=0 \quad \Longrightarrow \quad x=0 .
$$

We call $M(\cdot): \Omega \rightarrow R^{n \times n}$ a stochastic $R_{0}$ matrix [6] if

$$
x \geq 0, \quad M(\omega) x \geq 0, \quad x^{T} M(\omega) x=0, \quad \text { a.e. } \quad \Longrightarrow \quad x=0 .
$$


If $\Omega$ is a singleton, then $M(\omega)$ is an $R_{0}$ matrix. It is known that the $R_{0}$ function is a generalization of $A x+b$ with $A$ being an $R_{0}$ matrix [16]. Here we show that the stochastic $R_{0}$ function is a generalization of $M(\omega) x+q(\omega)$ with $M(\cdot)$ being a stochastic $R_{0}$ matrix.

Proposition 2.3 Let $F$ be an affine function of $x$ for any $\omega \in \Omega$ defined by (2). Then, $F$ is a stochastic $R_{0}$ function on $R_{+}^{n}$ if and only if $M(\cdot)$ is a stochastic $R_{0}$ matrix.

Proof ('If' part) Suppose on the contrary that $F$ is not a stochastic $R_{0}$ function on $R_{+}^{n}$, then there exists a sequence $\left\{x^{k}\right\} \subset R_{+}^{n}$ satisfying (6) in Definition 2.2, such that

$$
\mathcal{P}\left\{\omega: \limsup _{k \rightarrow \infty} \min \left(x_{i}^{k}, F_{i}\left(x^{k}, \omega\right)\right)=\infty\right\}=0, \quad \text { for all } i \in\langle 1, n\rangle .
$$

Let $x$ be any accumulation point of the bounded sequence $\left\{\frac{x^{k}}{\left\|x^{k}\right\|}\right\}$. Notice that $F\left(x^{k}, \omega\right)=M(\omega) x^{k}+q(\omega)$ for all $\omega \in \Omega$. We have

$$
\|x\|=1, \quad x \geq 0, \quad M(\omega) x \geq 0, \quad x^{T} M(\omega) x=0, \quad \text { a.e. }
$$

This contradicts $M(\cdot)$ being a stochastic $R_{0}$ matrix.

('Only if' part) Suppose on the contrary that $M(\cdot)$ is not a stochastic $R_{0}$ matrix, then there exists a vector $x \in R^{n}$ satisfying

$$
0 \neq x \geq 0, \quad M(\omega) x \geq 0, \quad x^{T} M(\omega) x=0 \quad \text { a.e. }
$$

Note that $q_{i}(\omega)<\infty$ a.e. by using Assumption A1 that $E\left[\|F(0, \omega)\|^{2}\right]=E\left[\|q(\omega)\|^{2}\right]$ $<\infty$. Define a sequence $\left\{x^{k}\right\}$ where $x^{k}=k x$ for $k=1,2, \ldots$ From $M(\omega) x^{k} \geq 0$, we have that $-F\left(x^{k}, \omega\right)=-\left(M(\omega) x^{k}+q(\omega)\right) \leq-q(\omega)$ for any $k$ and

$$
\limsup _{k \rightarrow \infty}\left\|\left(-F\left(x^{k}, \omega\right)\right)_{+}\right\| \leq\left\|(-q(\omega))_{+}\right\| \leq\|q(\omega)\|<\infty, \quad \text { a.e. }
$$

Hence, $\left\{x^{k}\right\} \subset R_{+}^{n}$ satisfies condition (6).

For an index $i \in\langle 1, n\rangle$ such that $x_{i}=0$, we have

$$
\min \left(x_{i}^{k}, F_{i}\left(x^{k}, \omega\right)\right)=\min \left(0, F_{i}\left(x^{k}, \omega\right)\right) \leq 0, \quad \omega \in \Omega .
$$

For an index $i \in\langle 1, n\rangle$ such that $x_{i}>0$, we have $(M(\omega) x)_{i}=0$ a.e., which implies $F_{i}\left(x^{k}, \omega\right)=k(M(\omega) x)_{i}+q_{i}(\omega)=q_{i}(\omega)$ a.e. Therefore,

$$
\mathcal{P}\left\{\omega: \limsup _{k \rightarrow \infty} \min \left(x_{i}^{k}, F_{i}\left(x^{k}, \omega\right)\right)=\infty\right\}=0,
$$

which contradicts $F$ being a stochastic $R_{0}$ function.

Now, we investigate the relation between $F$ being a stochastic $R_{0}$ function and the coercivity of the objective function $f_{1}$ in the ERM formulation.

Theorem 2.1 Suppose that $F$ is equicoercive on $R_{+}^{n}$. Then, $f_{1}$ is coercive on $R_{+}^{n}$ if and only if $F$ is a stochastic $R_{0}$ function on $R_{+}^{n}$. 
Proof ('If' part) Suppose on the contrary that $f_{1}$ is not coercive on $R_{+}^{n}$. Thus, there exists a sequence $\left\{x^{k}\right\} \subset R_{+}^{n}$ with $\left\|x^{k}\right\| \rightarrow \infty$ and a constant $a \in R_{+}$such that

$$
f_{1}\left(x^{k}\right) \leq a, \quad \forall k
$$

First, consider the case that $\left\{x^{k}\right\}$ does not satisfy (6). Thus, there exists $i \in\langle 1, n\rangle$ such that $\mathcal{P}\left\{\omega: \lim \sup _{k \rightarrow \infty}\left(-F_{i}\left(x^{k}, \omega\right)\right)_{+}=\infty\right\}>0$, and hence there are $\bar{\omega} \in$ $\operatorname{supp} \Omega$ and a subsequence $\left\{x^{\tilde{k}_{j}}\right\} \subseteq\left\{x^{k}\right\}$ such that $\lim _{\tilde{k}_{j} \rightarrow \infty}\left(-F_{i}\left(x^{\tilde{k}_{j}}, \bar{\omega}\right)\right)_{+}=\infty$. By the assumption that $F$ is equicoercive on $R_{+}^{n}$, there exists $\left\{x^{k_{j}}\right\} \subseteq\left\{x^{\tilde{k}_{j}}\right\}$ such that $\mathcal{P}\left\{\omega: \lim _{k_{j} \rightarrow \infty}\left(-F_{i}\left(x^{k_{j}}, \omega\right)\right)_{+}=\infty\right\}>0$. Let

$$
\Omega_{1}:=\left\{\omega: \lim _{k_{j} \rightarrow \infty} \min \left(x_{i}^{k_{j}}, F_{i}\left(x^{k_{j}}, \omega\right)\right)=-\infty\right\}
$$

Then, $\mathcal{P}\left\{\Omega_{1}\right\}>0$. By the Fatou lemma [17],

$$
E_{\Omega_{1}}\left[\liminf _{k_{j} \rightarrow \infty}\left(\min \left(x_{i}^{k_{j}}, F_{i}\left(x^{k_{j}}, \omega\right)\right)\right)^{2}\right] \leq \liminf _{k_{j} \rightarrow \infty} E_{\Omega_{1}}\left[\left(\min \left(x_{i}^{k_{j}}, F_{i}\left(x^{k_{j}}, \omega\right)\right)\right)^{2}\right] .
$$

Since $\liminf _{k_{j} \rightarrow \infty}\left(\min \left(x_{i}^{k_{j}}, F_{i}\left(x^{k_{j}}, \omega\right)\right)\right)^{2}=\infty$ on $\Omega_{1}$ and $\mathcal{P}\left\{\Omega_{1}\right\}>0$, the left-hand side of the above inequality is infinite. Hence,

$$
\liminf _{k_{j} \rightarrow \infty} E_{\Omega_{1}}\left[\left(\min \left(x_{i}^{k_{j}}, F_{i}\left(x^{k_{j}}, \omega\right)\right)\right)^{2}\right]=\infty
$$

Moreover, it is easy to find

$$
\begin{aligned}
f_{1}\left(x^{k_{j}}\right) & =E\left[\left\|\Phi\left(x^{k_{j}}, F\left(x^{k_{j}}, \omega\right)\right)\right\|^{2}\right] \\
& \geq E_{\Omega_{1}}\left[\left(\min \left(x_{i}^{k_{j}}, F_{i}\left(x^{k_{j}}, \omega\right)\right)\right)^{2}\right] \rightarrow \infty, \quad \text { as } k_{j} \rightarrow \infty .
\end{aligned}
$$

This contradicts to the fact that $f_{1}\left(x^{k}\right) \leq a$ for $\forall k$. Thus $\left\{x^{k}\right\} \subset R_{+}^{n}$ must satisfy (6). According to Definition 2.2, we choose an index $i \in\langle 1, n\rangle$ such that $\mathcal{P}\left\{\omega: \lim \sup _{k \rightarrow \infty} \min \left(x_{i}^{k}, F_{i}\left(x^{k}, \omega\right)\right)=\infty\right\}>0$. Since $F$ is equicoercive on $R_{+}^{n}$, we get that there exists $\left\{x^{k_{j}}\right\} \subseteq\left\{x^{k}\right\}$ such that $\mathcal{P}\left\{\omega: \lim _{k_{j} \rightarrow \infty} \min \left(x_{i}^{k_{j}}, F_{i}\left(x^{k_{j}}, \omega\right)\right)=\right.$ $\infty\}>0$. Let

$$
\Omega_{2}:=\left\{\omega: \lim _{k_{j} \rightarrow \infty} \min \left(x_{i}^{k_{j}}, F_{i}\left(x^{k_{j}}, \omega\right)\right)=\infty\right\}
$$

Then $\mathcal{P}\left\{\Omega_{2}\right\}>0$. Again by using the Fatou lemma,

$$
f_{1}\left(x^{k_{j}}\right) \geq E_{\Omega_{2}}\left[\left(\min \left(x_{i}^{k_{j}}, F_{i}\left(x^{k_{j}}, \omega\right)\right)\right)^{2}\right] \rightarrow \infty, \quad \text { as } k_{j} \rightarrow \infty,
$$

which is a contradiction to $f_{1}\left(x^{k}\right) \leq a$ for $\forall k$.

('Only if' part) Suppose on the contrary that $F$ is not a stochastic $R_{0}$ function on $R_{+}^{n}$, then there exists a sequence $\left\{x^{k}\right\} \subset R_{+}^{n}$ satisfying (6), such that 


$$
\mathcal{P}\left\{\omega: \limsup _{k \rightarrow \infty} \min \left(x_{i}^{k}, F_{i}\left(x^{k}, \omega\right)\right)=\infty\right\}=0, \quad \text { for any } i \in\langle 1, n\rangle .
$$

We then declare that there must exist constants $\underline{c}$ and $\bar{c}$ such that, for any $i \in\langle 1, n\rangle$,

$$
\underline{c} \leq \min \left(x_{i}^{k}, F_{i}\left(x^{k}, \omega\right)\right) \leq \bar{c}, \quad \forall \omega \in \operatorname{supp} \Omega .
$$

Suppose on the contrary that (8) is not true; then, there exist $\left\{\omega^{k}\right\} \subseteq \operatorname{supp} \Omega$ and $\hat{i} \in$ $\langle 1, n\rangle$ such that

$$
\limsup _{k \rightarrow \infty}\left(-F_{\hat{i}}\left(x^{k}, \omega^{k}\right)\right)_{+}=\infty \quad \text { or } \quad \limsup _{k \rightarrow \infty} \min \left(x_{\hat{i}}^{k}, F_{\hat{i}}\left(x^{k}, \omega^{k}\right)\right)=\infty .
$$

Hence there must exist a subsequence $\left\{x^{\tilde{k}_{j}}\right\} \subseteq\left\{x^{k}\right\}$ such that $\lim _{\tilde{k}_{j} \rightarrow \infty}\left(-F_{\hat{i}}\left(x^{\tilde{k}_{j}}\right.\right.$, $\left.\left.\omega^{\tilde{k}_{j}}\right)\right)_{+}=\infty$; or $\lim _{\tilde{k}_{j} \rightarrow \infty} \min \left(x_{\hat{i}}^{\tilde{k}_{j}}, F_{\hat{i}}\left(x^{\tilde{k}_{j}}, \omega^{\tilde{k}_{j}}\right)\right)=\infty$. By the assumption that $F$ is equicoercive on $R_{+}^{n}$, for the first case we know that there exists a subsequence $\left\{x^{k_{j}}\right\} \subseteq\left\{x^{\tilde{k}_{j}}\right\}$ such that $\mathcal{P}\left\{\omega: \lim _{k_{j} \rightarrow \infty}\left(-F_{\hat{i}}\left(x^{k_{j}}, \omega\right)\right)_{+}=\infty\right\}>0$, which contradicts to (6); For the second case, we know that $\mathcal{P}\left\{\omega: \lim _{k_{j} \rightarrow \infty} F_{\hat{i}}\left(x^{k_{j}}, \omega\right)=\infty\right\}>0$, which implies that

$$
\mathcal{P}\left\{\omega: \limsup _{k \rightarrow \infty} \min \left(x_{\hat{i}}^{k}, F_{\hat{i}}\left(x^{k}, \omega\right)\right)=\infty\right\}>0 .
$$

This contradicts (7). Therefore, (8) holds and we get

$$
\begin{aligned}
f_{1}\left(x^{k}\right) & =\sum_{i=1}^{n} E\left[\left(\min \left(x_{i}^{k}, F_{i}\left(x^{k}, \omega\right)\right)\right)^{2}\right] \\
& =\sum_{i=1}^{n} E_{\operatorname{supp} \Omega}\left[\left(\min \left(x_{i}^{k}, F_{i}\left(x^{k}, \omega\right)\right)\right)^{2}\right] \leq n(\max \{|\bar{c}|,|\underline{c}|\})^{2} .
\end{aligned}
$$

Notice that the sequence $\left\{x^{k}\right\} \subset R_{+}^{n}$ satisfies (6) and the sequence $\left\{f_{1}\left(x^{k}\right)\right\}$ is bounded. This contradicts to the coercivity of $f_{1}$ on $R_{+}^{n}$.

Remark 2.2 Following the proof of Theorem 2.1, we can see that if for every sequence $\left\{x^{k}\right\} \subset R_{+}^{n}$ satisfying $\lim _{k \rightarrow \infty}\left\|x^{k}\right\|=\infty$, there exists $i \in\langle 1, n\rangle$ and a subsequence $\left\{x^{k_{j}}\right\}$ such that

$$
\begin{aligned}
& \mathcal{P}\left\{\omega: \lim _{k_{j} \rightarrow \infty}\left(-F_{i}\left(x^{k_{j}}, \omega\right)\right)_{+}=\infty\right\}>0, \text { or } \\
& \mathcal{P}\left\{\omega: \lim _{k_{j} \rightarrow \infty} \min \left(x_{i}^{k_{j}}, F_{i}\left(x^{k_{j}}, \omega\right)\right)=\infty\right\}>0,
\end{aligned}
$$

then $F$ is a stochastic $R_{0}$ function and $f_{1}$ is coercive on $R_{+}^{n}$.

Similar results for the coercivity of $f$ defined by other NCP functions can be obtained by noticing their relations with $\phi_{1}$. In particular, from (4), we have the following corollary. 


\section{Corollary 2.1}

(i) Suppose that $F$ is equicoercive on $R_{+}^{n}$. Then, $f_{2}$ is coercive on $R_{+}^{n}$ if and only if $F$ is a stochastic $R_{0}$ function on $R_{+}^{n}$.

(ii) If $F$ is a stochastic $R_{0}$ function and equicoercive on $R_{+}^{n}$, then $f_{3}$ is coercive on $R_{+}^{n}$.

From Theorem 2.1 and Corollary 2.1, we obtain immediately the following corollary.

Corollary 2.2 If $F$ is a stochastic $R_{0}$ function and equicoercive on $R_{+}^{n}$, then the solution set of (3) defined by $\phi_{i}, i=1,2,3$ is nonempty and bounded.

\section{ERM-SNCP Model for TEP under Uncertainty}

Let $[\mathcal{N}, \mathcal{A}]$ represent a given transportation network, where $\mathcal{N}$ is the set of nodes, and $\mathcal{A}$ is the set of links. We use $\Omega \subseteq R^{m}$ to represent a set of random vectors. Each vector $\omega \in \Omega$, corresponding to one realization of stochastic factors such as weather, accidents, etc., is of given probability $\mathcal{P}$. For any realization $\omega \in \Omega$, let us denote

$\begin{array}{ll}\mathcal{I} & \text { the set of origin-destination (OD) pairs, } \\ \mathcal{R}_{i} & \text { the set of "available" routes, connecting OD pair } i \text { (which } \\ & \text { might, but not necessarily be all paths joining the OD pair), } \\ h_{r}(\omega) & \text { the flow on route } r, \\ \Delta & \text { the link-route incidence matrix of the network, } \\ \Gamma & \text { the OD pair-route incidence matrix of the network, } \\ u_{i}(\omega) & \text { the shortest travel cost function for OD pair } i, \\ d_{i}(\omega) & \text { the demand function for OD pair } i, \\ C_{r}(h(\omega), \omega) & \text { the travel cost function for route } r .\end{array}$

Moreover, let $\mathcal{R}=\bigcup_{i \in \mathcal{I}} \mathcal{R}_{i}$ and $u(\omega), d(\omega), h(\omega), C(h(\omega), \omega)$ represent the vector composed of $u_{i}(\omega), d_{i}(\omega), h_{r}(\omega), C_{r}(h(\omega), \omega)$ for $i \in \mathcal{I}, r \in \mathcal{R}$, respectively. It is clear that

$$
u, d: \Omega \rightarrow R_{+}^{|\mathcal{I}|}, \quad h: \Omega \rightarrow R_{+}^{|\mathcal{R}|}, \quad C: R_{+}^{|\mathcal{R}|} \times \Omega \rightarrow R_{+}^{|\mathcal{R}|} .
$$

Here, we suppose that the uncertain demand $d(\omega)$ is bounded for almost all $\omega \in \Omega$. We say that the network $[\mathcal{N}, \mathcal{A}]$ is strongly connected if for any OD pair $i \in \mathcal{I}$ there is at least one route joining the origin to the destination. Then each row of $\Gamma$ is a nonzero vector. Moreover, since one route connects only one OD pair, $\Gamma$ has full row-rank. The link-route incidence matrix $\Delta$ is deterministic for the given network.

In a congested network, drivers have the incentive to compete with each other for selecting the route with minimal travel cost, at a certain level of travel demand. The traffic equilibrium problem (TEP) has been used for transportation planning, which seeks for flow pattern with the equilibrium property that no driver may decrease his travel cost by unilaterally changing his route. It is the interaction between drivers that forms the stable flow pattern in the equilibrium state and such flow pattern is used by 
the administrator for predicting the traffic flow. For more details about TEP, we refer to [18].

The Wardrop equilibrium principle [9] for the genesis of the TEP states that in the equilibrium state, for any OD pair the travel cost on every used routes equals and any route needs higher travel cost will have no traffic flow. Application of the Wardrop equilibrium for the realization $\omega \in \Omega$ gives

$$
\begin{aligned}
C_{r}(h(\omega), \omega)-u_{i}(\omega) & \geq 0, \quad h_{r}(\omega) \geq 0, \\
\left(C_{r}(h(\omega), \omega)-u_{i}(\omega)\right) h_{r}(\omega) & =0, \quad i \in \mathcal{I}, r \in \mathcal{R}_{i} .
\end{aligned}
$$

Moreover, according to the demand conservation, we have

$$
\sum_{r \in \mathcal{R}_{i}} h_{r}(\omega)-d_{i}(\omega)=0
$$

which is equivalent to

$$
\begin{aligned}
\sum_{r \in \mathcal{R}_{i}} h_{r}(\omega)-d_{i}(\omega) & \geq 0, \quad u_{i}(\omega) \geq 0, \\
\left(\sum_{r \in \mathcal{R}_{i}} h_{r}(\omega)-d_{i}(\omega)\right) u_{i}(\omega) & =0, \quad i \in \mathcal{I}, r \in \mathcal{R}_{i},
\end{aligned}
$$

under some mild assumptions that would be expected to meet always in practice [10]. (9)-(10) is the NCP formulation of static TEP ([10] and [19]) for each fixed $\omega \in \Omega$. In particular, we can write (9)-(10) as

$$
x_{\omega} \geq 0, \quad F\left(x_{\omega}, \omega\right) \geq 0, \quad x_{\omega}^{T} F\left(x_{\omega}, \omega\right)=0, \quad \omega \in \Omega,
$$

where

$$
x_{\omega}=\left(\begin{array}{c}
h(\omega) \\
u(\omega)
\end{array}\right), \quad F\left(x_{\omega}, \omega\right)=\left(\begin{array}{c}
C(h(\omega), \omega)-\Gamma^{T} u(\omega) \\
\Gamma h(\omega)-d(\omega)
\end{array}\right) .
$$

The solution $x_{\omega}$ of (11) depends on an unknown realization $\omega$, which can only be predicted such as weather. It is interesting for the administrator to find a reliable flow pattern that is not far from optimal flow pattern $x_{\omega}$ given by (11). Such flow pattern may help for future planning. In other words, we wish that there was a deterministic vector $x \in R^{|\mathcal{R}|+|\mathcal{I}|}$ satisfying the SNCP

$$
x \geq 0, \quad F(x, \omega) \geq 0, \quad x^{T} F(x, \omega)=0, \quad \omega \in \Omega,
$$

where

$$
x=\left(\begin{array}{l}
h \\
u
\end{array}\right), \quad F(x, \omega)=\left(\begin{array}{c}
C(h, \omega)-\Gamma^{T} u \\
\Gamma h-d(\omega)
\end{array}\right) .
$$

However, in general, we can not find such vector $x$ that is the equilibria for any random vector $\omega \in \Omega$. We have to consider a deterministic formulation of (12) such as the $\mathrm{EV}$ formulation $\operatorname{NCP}(E[F(x, \omega)])$ and the ERM formulation (3). The ERM 
formulation provides a solution $x^{*}=\left(h^{*}, u^{*}\right)$ that minimizes expected violation of the equilibrium (9)-(10), and represents the most likely equilibrium flow pattern $h^{*}$ and travel cost $u^{*}$ before we know the realization of uncertain factors. In general, we do not have $x^{*}=x_{\omega}$ for all $\omega \in \Omega$. The violation of $x^{*}$ to (9)-(10) is natural, which means $x^{*}$ has error to $x_{\omega}$ for some $\omega \in \Omega$.

In what follows, we let $v_{a}$ be the travel flow on link $a$, and $v$ be the link travel flow vector with components $v_{a}, a \in \mathcal{A}$. We use the function $t_{a}(v, \omega)$ to denote the travel time on link $a$, and $t(v, \omega)$ for the link travel time vector with components $t_{a}(v, \omega)$, $a \in \mathcal{A}$. Clearly, the link travel flow vector $v$ and the route travel flow vector $h$ have the following relationship:

$$
v=\Delta h
$$

It is pointed out in [19] that in many cases the travel cost function is nonadditive, which may rise from a variety of transportation polices, nonlinear valuation of travel time, etc. In this paper, we add random factors $\omega$ to the general nonadditive travel cost function suggested in [19] as

$$
C(h, \omega)=\eta_{1} \Delta^{T} t(\Delta h, \omega)+g\left(\Delta^{T} t(\Delta h, \omega)\right)+\Lambda(h, \omega),
$$

where $\eta_{1}>0$ is the time-based operating costs factor, $g: R_{+}^{|\mathcal{R}|} \rightarrow R_{+}^{|\mathcal{R}|}$ is the translation function converting time $t$ to money, and $\Lambda$ is the perturbed financial cost function (e.g., distance-based operating costs such as maintenance). We call (14) the perturbed general nonadditive travel cost function. We suppose the following assumption on the travel time function and the travel cost function holds.

Assumption A2 There exists a subset $\hat{\Omega} \subseteq \Omega$ with $\mathcal{P}\{\hat{\Omega}\}>0$, such that, for any $\omega \in \hat{\Omega}$,

(i) the travel cost function $C_{r}(h, \omega)$ on each route is a nondecreasing function of flow $h$, and finite for any fixed $h$;

(ii) the travel time function $t_{a}(v, \omega)$ on each link is a nondecreasing function of flow $v$, finite for any fixed $v$, and coercive with flow on the link $v_{a}$, i.e., $t_{a}(v, \omega) \rightarrow \infty$ if $v_{a} \rightarrow \infty$.

Assumption A2 holds in various perturbed travel cost and travel time functions used in practice. For instance, let the perturbed travel cost function be

$$
C(h, \omega)=\left(\Delta^{T} t(\Delta h, \omega)\right)^{2},
$$

and let the travel time function $t$ be

$$
t_{a}(v, \omega):=(K(\omega) v+k(\omega))_{a}, \quad a \in \mathcal{A},
$$

where $K(\omega) \in R_{+}^{|\mathcal{A}| \times|\mathcal{A}|}$ has positive diagonal elements and $k(\omega) \in R_{+}^{|\mathcal{A}|}$ for any $\omega \in$ $\Omega$. For a fixed $\omega$, this is the simple affine travel time function used in [11], where it is said that $K(\omega)$ is in general a positive semi-definite matrix. 
Proposition 3.1 Suppose that the network $[\mathcal{N}, \mathcal{A}]$ is strongly connected and that Assumption A2 holds; then, $F$ in (13) is a stochastic $R_{0}$ function on $R_{+}^{n}$.

Proof For any infinite sequence $\left\{x^{k}\right\} \subset R_{+}^{n}$ satisfying (6), let us choose a subsequence $\left\{x^{k_{j}}\right\} \subseteq\left\{x^{k}\right\}$ such that $x_{l}^{k_{j}} \rightarrow \infty$ as $k_{j} \rightarrow \infty$ for some $l \in\langle 1, n\rangle$. Recall that $n=|\mathcal{R}|+|\mathcal{I}|$.

If $l \in\langle 1,|\mathcal{R}|\rangle$, we have $h_{l}^{k_{j}} \rightarrow \infty$ as $k_{j} \rightarrow \infty$. Notice that $\Delta_{a l}=1$ for any link $a$ on route $l$, thus $\left(\Delta h^{k_{j}}\right)_{a} \geq h_{l}^{k_{j}} \rightarrow \infty$ as $k_{j} \rightarrow \infty$. This indicates that $t_{a}\left(\Delta h^{k_{j}}, \omega\right) \rightarrow$ $\infty$ as $k_{j} \rightarrow \infty$ for $\hat{\Omega} \subseteq \Omega$ with $\mathcal{P}\{\hat{\Omega}\}>0$ by (ii) of Assumption A2. Hence, for any $\omega \in \hat{\Omega}$,

$$
C_{l}\left(h^{k_{j}}, \omega\right) \geq \eta_{1}\left(\Delta^{T} t\left(\Delta h^{k_{j}}, \omega\right)\right)_{l} \geq \eta_{1} \Delta_{a l} t_{a}\left(\Delta h^{k_{j}}, \omega\right)=\eta_{1} t_{a}\left(\Delta h^{k_{j}}, \omega\right) \rightarrow \infty,
$$

as $k_{j} \rightarrow \infty$. If $\left\{\left(\Gamma^{T} u^{k_{j}}\right)_{l}\right\}$ is bounded, then $F_{l}\left(x^{k_{j}}, \omega\right)=\left(C\left(h^{k_{j}}, \omega\right)-\Gamma^{T} u^{k_{j}}\right)_{l} \rightarrow \infty$ as $k_{j} \rightarrow \infty$ for $\omega \in \hat{\Omega}$. From Definition 2.2, we find that $F$ is a stochastic $R_{0}$ function, since

$$
\mathcal{P}\left\{\omega: \lim _{k_{j} \rightarrow \infty} \min \left(x_{l}^{k_{j}}, F_{l}\left(x^{k_{j}}, \omega\right)\right)=\infty\right\} \geq \mathcal{P}\{\hat{\Omega}\}>0
$$

Otherwise, we have $\left(\Gamma^{T} u^{k_{j}}\right)_{l} \rightarrow \infty$ as $k_{j} \rightarrow \infty$. This implies the existence of $i \in \mathcal{I}$ such that $\Gamma_{i l}=1$ and $u_{i}^{k_{j}} \rightarrow \infty$. Thus, for any $\omega \in \hat{\Omega}$,

$$
\left(\Gamma h^{k_{j}}-d(\omega)\right)_{i} \geq \Gamma_{i l} h_{l}^{k_{j}}-d_{i}(\omega) \rightarrow \infty, \quad \text { as } k_{j} \rightarrow \infty .
$$

Hence, $F$ is a stochastic $R_{0}$ function by noticing the expression of $F$ in (13) and

$$
\mathcal{P}\left\{\omega: \lim _{k_{j} \rightarrow \infty} \min \left(u_{i}^{k_{j}},\left(\Gamma h^{k_{j}}-d(\omega)\right)_{i}\right)=\infty\right\} \geq \mathcal{P}\{\hat{\Omega}\}>0 .
$$

Now, we consider $l \in\langle|\mathcal{R}|+1, n\rangle$ and $\left\{h^{k_{j}}\right\}$ is bounded. Then, we have $u_{i}^{k_{j}} \rightarrow \infty$ as $k_{j} \rightarrow \infty$ for some $i \in \mathcal{I}$. Since the network is strongly connected, there exists $\Gamma_{i r}=1$ for any route $r$ connecting OD pair $i$. Thus, we get

$$
\left(\Gamma^{T} u^{k_{j}}\right)_{r} \geq \Gamma_{i r} u_{i}^{k_{j}}=u_{i}^{k_{j}} \rightarrow \infty \quad \text { as } k_{j} \rightarrow \infty .
$$

Moreover, $\left\{C_{r}\left(h^{k_{j}}, \omega\right)\right\}$ is bounded for $\omega \in \hat{\Omega}$ by using (i) of Assumption A2 and the fact that $\left\{h^{k_{j}}\right\}$ is bounded. Hence $\left(C\left(h^{k_{j}}, \omega\right)-\Gamma^{T} u^{k_{j}}\right)_{r} \rightarrow-\infty$ as $k_{j} \rightarrow \infty$ for $\omega \in \hat{\Omega}$. From the expression of $F$ in (13), we get

$$
\lim _{k_{j} \rightarrow \infty}\left\|\left(-F\left(x^{k_{j}}, \omega\right)\right)_{+}\right\| \geq \lim _{k_{j} \rightarrow \infty}\left|-\left(C\left(h^{k_{j}}, \omega\right)-\Gamma^{T} u^{k_{j}}\right)_{r}\right|=\infty \quad \text { for } \omega \in \hat{\Omega}
$$

where $\mathcal{P}\{\hat{\Omega}\}>0$. This is impossible, since $\left\{x^{k}\right\}$ satisfies (6).

Hence, $F$ is a stochastic $R_{0}$ function on $R_{+}^{n}$.

Remark 3.1 It is easy to see that $F$ in fact satisfies the condition in Remark 2.2. Hence the objective function $f_{1}$ is coercive on $R_{+}^{n}$, and the solution set of the ERM formulation for $\operatorname{SNCP}(F(x, \omega))$ is nonempty and bounded. 


\section{Evaluation of the ERM-SNCP Model for TEP under Uncertainty}

In this section, we report computational experiments that compare the proposed ERM-SNCP model with EV-SNCP model through a simple example of TEP under uncertainty. We begin with definitions of performance measure which evaluate the quality for a flow pattern such as reliability, unfairness, and total travel cost.

The reliability ([4-6] and [20]) concerns the safety of a flow pattern, that is, the probability to be feasible. For a flow pattern $h$, its reliability is defined by

$$
\operatorname{rel}(h):=\mathcal{P}\left\{\omega:(\Gamma h-d(\omega))_{i} \geq 0, i=|\mathcal{R}|+1, \ldots, n\right\}
$$

Notice that $(\Gamma h-d(\omega))_{i} \geq 0$ manifests that the demand for OD pair $i \in \mathcal{I}$ and $\omega \in \Omega$ can be delivered in the traffic flow pattern $h$.

For a flow pattern $h$, the expected ratio of the delivered demand to the total demand of the system is defined by

$$
\operatorname{dr}(h):=E\left[\frac{1}{|\mathcal{I}|} \sum_{i \in \mathcal{I}} \frac{\min \left((\Gamma h)_{i}, d_{i}(\omega)\right)}{d_{i}(\omega)}\right] .
$$

Clearly $0 \leq \operatorname{dr}(h) \leq 1$ and the nearer $\operatorname{dr}(h)$ is to 1 , the more feasibility the solution earns in practice.

For each fixed $\omega \in \Omega$, the Wardrop equilibria reflects the fairness to all users with the same OD pair, since the travel cost for each used route connecting the same OD pair is equal and less than any unused route. However, for the uncertain case, the travel cost for any flow pattern connecting the same OD pair is not necessarily the same. For a fixed $\omega \in \Omega$, the unfairness of a feasible flow pattern for an OD pair $i \in \mathcal{I}$ [21] is measured by

$$
C_{i}^{\text {unfair }}(h, \omega)=\frac{C_{i}^{\max }(h, \omega)}{C_{i}^{\min }(h, \omega)},
$$

where $C_{i}^{\max }(h, \omega)$ and $C_{i}^{\min }(h, \omega)$ are the largest and smallest travel cost of routes being used, which connect OD pair $i$. Thus, the expected unfairness of the decision for the whole system under uncertainty is defined by

$$
\operatorname{unf}(h):=E\left[\frac{1}{|\mathcal{I}|} \sum_{i \in \mathcal{I}} C_{i}^{\text {unfair }}(h, \omega)\right]=E\left[\frac{1}{|\mathcal{I}|} \sum_{i \in \mathcal{I}} \frac{C_{i}^{\max }(h, \omega)}{C_{i}^{\min }(h, \omega)}\right]
$$

For a flow pattern $h$, the corresponding expected travel cost is defined by

$$
\operatorname{tc}(h):=E\left[h^{T} C(h, \omega)\right] .
$$

We use a simple example to illustrate the ERM-SNCP model for the traffic equilibrium under uncertainty.

Example 4.1 The transportation network shown in Fig. 1 is adopted from [22], which has 13 nodes, 19 links and 4 OD pairs $(1 \rightarrow 2,1 \rightarrow 3,4 \rightarrow 2,4 \rightarrow 3)$, with the network characters $t_{a}^{0}$ and $c_{a}^{0}$. 
Fig. 1 An example network

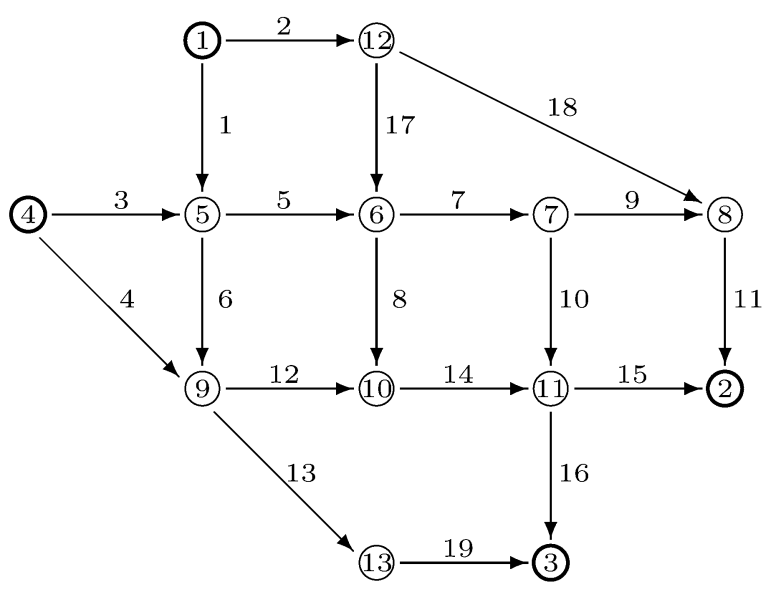

We suppose that the perturbed travel cost function is defined as

$$
C(h, \omega)=\Delta^{T} t(\Delta h, \omega), \quad \omega \in \Omega,
$$

where the perturbed travel time function, derived from the Bureau of Public Road link travel time function (1964), can be written as

$$
t_{a}(v, \omega):=t_{a}^{0}\left(1+0.15\left(\frac{v_{a}}{c_{a}(\omega)}\right)^{4}\right), \quad a \in \mathcal{A} .
$$

Here $t_{a}^{0}>0$ is the travel time in the network without congestion, and $c_{a}(\omega) \geq 0$ represents perturbed link capacity with $\mathcal{P}\left\{\omega: c_{a}(\omega)>0\right\}>0$ for all $a \in \mathcal{A}$. For any fixed $\omega$, it is a separable function, i.e., for each link, the travel time depends only on the travel flow and capacity of this link.

Case 1. Suppose that $c(\omega) \equiv c^{0}$ and $d(\omega)=\omega=\left(\omega_{1}, \omega_{2}, \omega_{3}, \omega_{4}\right)$, where $\omega_{1}, \omega_{2}, \omega_{3}$, $\omega_{4}$ follow the independent truncated normal distributions, respectively,

$$
\begin{array}{ll}
\omega_{1} \sim 300 \leq N(400,2500) \leq 500, & \omega_{2} \sim 600 \leq N(800,2500) \leq 1000, \\
\omega_{3} \sim 400 \leq N(600,2500) \leq 800, & \omega_{4} \sim 100 \leq N(200,900) \leq 300 .
\end{array}
$$

Case 2. Based on case 1, we suppose that some great changes of capacity of the link $a=5$ may happen due to the weather and road condition, as

$$
\mathcal{P}\left\{\omega: c_{5}(\omega) \equiv \frac{1}{4} c_{5}^{0}\right\}=\frac{1}{2}, \quad \mathcal{P}\left\{\omega: c_{5}(\omega) \equiv c_{5}^{0}\right\}=\frac{1}{2} .
$$

Case 3. Based on case 1, we extend the range of $\omega_{1}, \omega_{2}$ as

$$
\omega_{1} \sim 200 \leq N(400,2500) \leq 600, \quad \omega_{2} \sim 400 \leq N(800,2500) \leq 1200 .
$$

Let $x_{\mathrm{EV}}$ and $x_{\mathrm{ERM}}$ be the solutions of the EV and the ERM formulations of the SNCP (12), respectively. In Table 1, we report the computation results for the performance measure (15)-(18) as well as the number of used routes $\operatorname{nr}(h)$. Here, a used 
Table 1 Reliability, unfairness and total travel cost of $x_{\mathrm{EV}}$ and $x_{\mathrm{ERM}}$

\begin{tabular}{llll}
\hline$x_{\mathrm{EV}}$ & Case 1 & Case 2 & Case 3 \\
\hline Reliability rel $(h)$ & 0.0623 & 0.0623 & 0.0626 \\
Delivered rate $\operatorname{dr}(h)$ & $93.24 \%$ & $93.24 \%$ & $91.22 \%$ \\
Unfairness unf $(h)$ & 1.25 & 1.56 & 1.25 \\
Total travel cost tc $(h)$ & $7.93 \mathrm{e}+4$ & $8.47 \mathrm{e}+4$ & $7.93 \mathrm{e}+4$ \\
Num. of used routes $\operatorname{nr}(h)$ & 7 & 7 & 7 \\
\hline$x_{\text {ERM }}$ & Case 1 & Case 2 & Case 3 \\
\hline Reliability rel $(h)$ & 0.5285 & 0.4586 & 0.5405 \\
Delivered $\operatorname{rate} \operatorname{dr}(h)$ & $99.41 \%$ & $99.14 \%$ & $99.25 \%$ \\
Unfairness unf $(h)$ & 1.38 & 1.73 & 1.45 \\
Total travel cost tc $(h)$ & $1.10 \mathrm{e}+5$ & $1.21 \mathrm{e}+5$ & $1.39 \mathrm{e}+5$ \\
Num. of used $\operatorname{routes} \operatorname{nr}(h)$ & 19 & 16 & 19 \\
\hline
\end{tabular}

Fig. 2 Travel flow pattern of ERM-SNCP in case 1

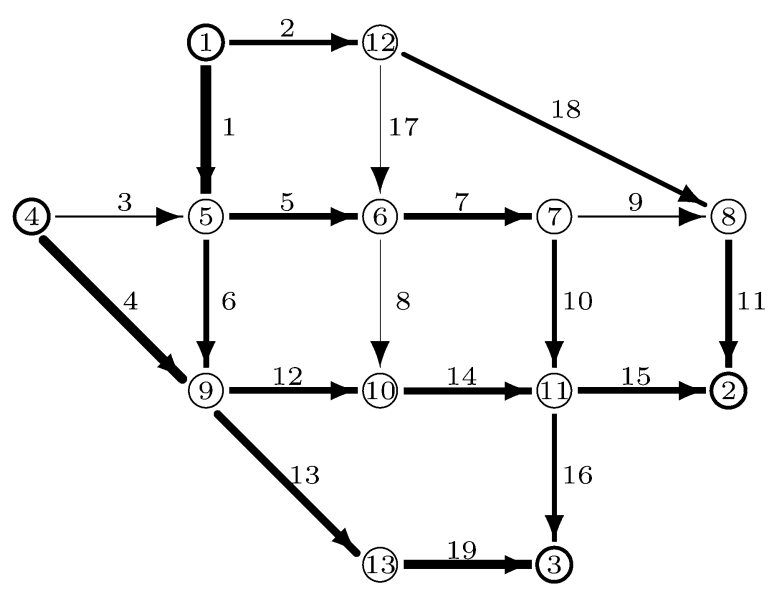

route refers to the route that has flow $h_{r} \geq 0.0001$. The results are the average of 100 simulations for $\Omega=\left\{\omega^{1}, \omega^{2}, \ldots, \omega^{1000}\right\}$. The sample points were obtained by the Monte-Carlo method. Figures 2-4 show the travel flow pattern of the ERM-SNCP model for the three cases, respectively. Notice that the width of each link in Figs. 2-4 is proportional to the amount of travel flow on this link.

Preliminary numerical results of traffic equilibrium problems under uncertainty show that the flow pattern drawing from a solution $x_{\text {ERM }}$ of the ERM formulation has higher reliability and delivered rate than the EV formulation. On the other hand, the EV-SNCP formulation has lower unfairness and total travel cost than the ERM formulation. This phenomenon can be explained as follows. The EV formulation seeks equilibria with the expected value of the travel cost function and travel demand. The ERM formulation minimizes the violation (residual) of the equilibrium for all $\omega \in \Omega$. Hence the ERM formulation has higher reliability and delivered rate than the EV for- 
Fig. 3 Travel flow pattern of ERM-SNCP in case 2
Fig. 4 Travel flow pattern of ERM-SNCP in case 3
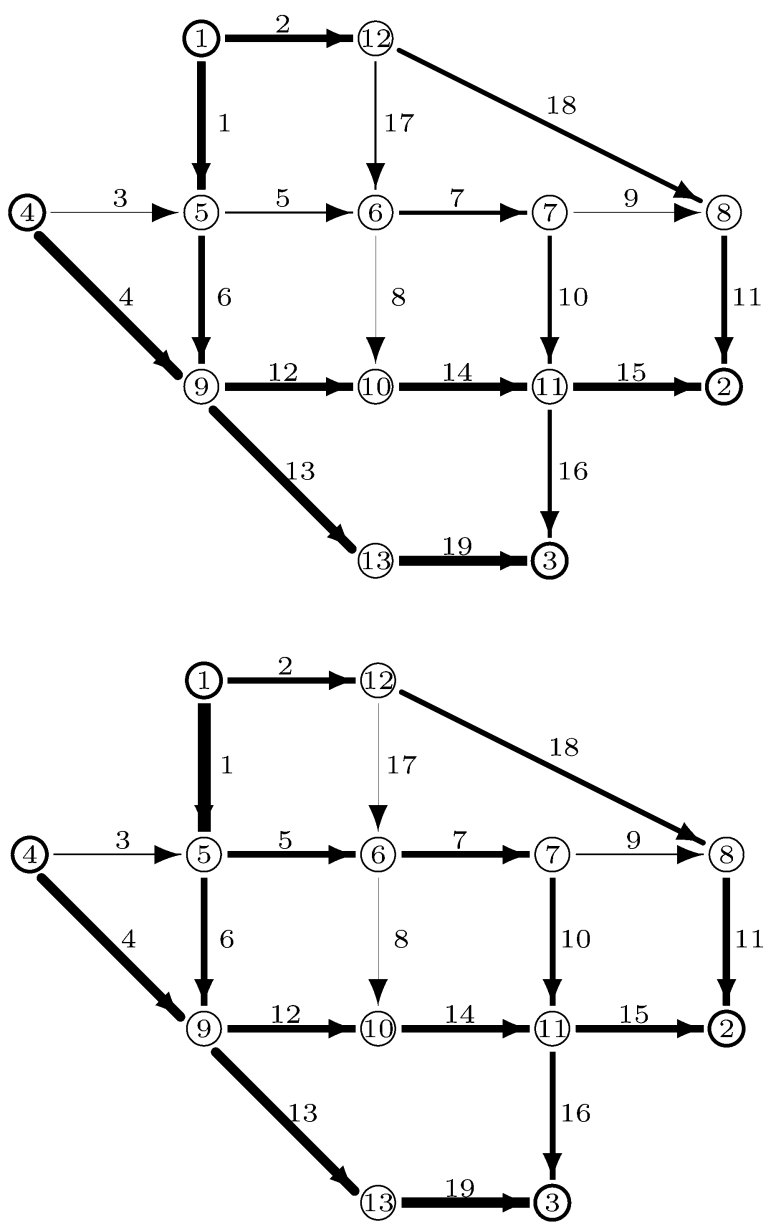

mulation. Since the ERM flow pattern delivers much more vehicles, its cost is higher than the EV flow pattern. Moreover, the unfairness of each flow pattern is defined on the routes being used, and the ERM flow pattern uses more routes than the EV one. This makes ERM flow pattern has higher unfairness than the EV formulation. Therefore, the EV formulation is recommended to administrators who prefer low cost, and the ERM formulation is recommended to administrators who want a reliable travel flow pattern which minimizes the expected violation of the equilibrium.

\section{References}

1. Cottle, R.W., Pang, J.S., Stone, R.E.: The Linear Complementarity Problem. Academic Press, Boston (1992)

2. Facchinei, F., Pang, J.S.: Finite-Dimensional Variational Inequalities and Complementarity Problem, I and II. Springer, New York (2003)

3. Lin, G.H., Fukushima, M.: New reformulations for stochastic nonlinear complementarity problems. Optim. Methods Softw. 21, 551-564 (2006) 
4. Chen, X., Fukushima, M.: Expected residual minimization method for stochastic linear complementarity problems. Math. Oper. Res. 30, 1022-1038 (2005)

5. Chen, X., Zhang, C., Fukushima, M.: Robust solution of monotone stochastic linear complementarity problems. Math. Program. (2007) online

6. Fang, H., Chen, X., Fukushima, M.: Stochastic $R_{0}$ matrix linear complementarity problems. SIAM J. Optim. 18, 482-506 (2007)

7. Gürkan, G., Özge, A.Y., Robinson, S.M.: Sample-path solution of stochastic variational inequalities. Math. Program. 84, 313-333 (1999)

8. Tseng, P.: Growth behavior of a class of merit functions for the nonlinear complementarity problem. J. Optim. Theory Appl. 89, 17-37 (1996)

9. Wardrop, J.G.: Some theoretical aspects of road traffic research. Proc. ICE Part II 1, 325-378 (1952)

10. Aashtiant, H.Z., Magnanti, T.L.: Equilibria on a congested transportation network. SIAM J. Algebr. Discrete Methods 2, 213-226 (1981)

11. Daffermos, S.: Traffic equilibrium and variational inequalities. Transp. Sci. 14, $42-54$ (1980)

12. Fukushima, M.: The primal Douglas-Rachford splitting algorithm for a class of monotone mapping with application to the traffic equilibrium problem. Math. Program. 72, 1-15 (1996)

13. Fernando, O., Nichlàs, E.S.: Robust Wardrop equilibrium. Technical Report (2006). See website http: //illposed.usc.edu./ fordon/docs/rwe.pdf

14. Ruszcyǹski, A., Shapiro, A. (eds.): Stochastic Programming. Handbooks in OR \& MS, vol. 10. NorthHolland, Amsterdam (2003)

15. Lin, G.H., Chen, X., Fukushima, M.: New restricted NCP functions and their applications to stochastic NCP and stochastic MPEC. Optimization 15, 641-653 (2007)

16. Chen, B.: Error bounds for $R_{0}$-type and monotone nonlinear complementarity problems. J. Optim. Theory Appl. 108, 297-316 (2001)

17. Chung, K.L.: A Course in Probability Theory, 2nd edn. Academic Press, New York (1974)

18. Patriksson, M.: Traffic Assignment Problems-Models and Methods. VSP, Utrecht (1994)

19. Gabriel, S.A., Bernstein, D.: The traffic equilibrium problem with nonadditive path costs. Trans. Sci. 31, 337-348 (1997)

20. Kall, P., Wallace, S.W.: Stochastic Programming. Wiley, New York (1994)

21. Jahn, O., Möhring, R.H., Schulz, A.S., Stier-Moses, N.E.: System-optimal routing of traffic flows with user constraints in networks with congestion. Oper. Res. 53, 600-616 (2005)

22. Yin, Y.F., Madanat, S.M., Lu, X.Y., Kuhn, K.D.: Robust improvement schemes for road networks under demand uncertainty. In: Proceedings of TRB 84th Annual Meeting, Washington (2005) 